\title{
TRIM3 inhibits P53 signaling in breast cancer cells
}

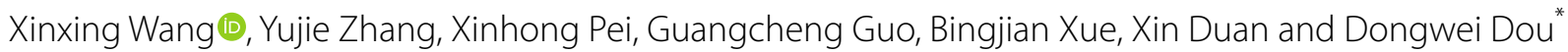

\begin{abstract}
Background: Beast cancer is the most common women cancer worldwide, while two third of them are ER alpha positive breast cancer. Among the ER alpha positive breast cancer, about 80\% are P53 wild type, indicating the potential tumor suppression role in ER alpha positive breast cancer. Since P53 is an important safeguard to inhibit cell malignant transformation, reactivating P53 signaling could a plausible approach to treat breast cancer.

Methods: TRIM3 protein levels were measured by western blot, while the P53 classical target genes were measured by real-time PCR. WST1 assay were used to measure cell proliferation, while cleaved caspase-3 was used to detect cell apoptosis. Protein stability and ubiquitin assay were used to detect the P53 protein ubiquitin and stability. The immuno-precipitation assays were used to detect the protein interactions. Immuno-staining was used to detect the protein localization of P53 and TRIM3, while the ubiquitin-based immuno-precipitation assays were used to detect the specific ubiquitination manner of P53.

Results: In our study, we identified TRIM3 as an endogenous inhibitor for P53 signaling. TRIM3 depletion inhibited breast cancer cell proliferation and promoted apoptosis. In addition, TRIM3 depletion increased P53 protein level in breast cancer cell. Further investigation showed that TRIM3 could associate with P53 and promote P53 K48-linked ubiquitination and degradation.
\end{abstract}

Conclusion: Our study identified a novel post-translational modification mechanism between TRIM3 and P53. TRIM3 depletion or blockage could be a promising strategy to rescue P53 signaling and inhibit breast cancer progression.

Keywords: TRIM3, P53, Breast cancer, Ubiquitin

\section{Highlights}

1. TRIM3 facilitates breast cancer cell growth and antiapotosis.

2. TRIM3 inhibits P53 protein and its signaling activity.

3. TRIM3 interacts with P53 and promotes P53 K48linked ubiquitination and degradation.
*Correspondence: dongwei.dou@gmail.com

Department of Breast Surgery, The First Affiliated Hospital of Zhengzhou University, Henan Province 450052, Zhengzhou, People's Republic of China

\section{Background}

Breast cancer is one of the most common malignancies in women worldwide. According to the latest statistical reports, more than 1.6 million breast cancer new cases are diagnosed each year, which account for $20 \%$ of all women cancers [1]. According to the molecular subtype classification, breast cancer can be classified into five groups: Luminal A type, Luminal B type, normal-like, HER2 type and basal-like [2]. Both Luminal A and B types are estrogen receptor alpha positive, which accounts for $70 \%$ of all breast cancers. Besides surgical treatment, the adjuvant therapy, such as endocrine therapy, is the most common treatment for luminal type of breast cancer patients [3]. Even the luminal type breast cancer patients could benefit from endocrine therapy, many patients will

(c) The Author(s) 2020. This article is licensed under a Creative Commons Attribution 4.0 International License, which permits use, sharing, adaptation, distribution and reproduction in any medium or format, as long as you give appropriate credit to the original author(s) and the source, provide a link to the Creative Commons licence, and indicate if changes were made. The images or other third party material in this article are included in the article's Creative Commons licence, unless indicated otherwise in a credit line to the material. If material is not included in the article's Creative Commons licence and your intended use is not permitted by statutory regulation or exceeds the permitted use, you will need to obtain permission directly from the copyright holder. To view a copy of this licence, visit http://creativeco mmons.org/licenses/by/4.0/. The Creative Commons Public Domain Dedication waiver (http://creativecommons.org/publicdomain/ zero/1.0/) applies to the data made available in this article, unless otherwise stated in a credit line to the data. 
develop tamoxifen resistance [4]. Thus it is urgent and necessary to develop more novel therapeutics targets for luminal type breast cancer patients.

The P53 protein was firstly found in 1979 and was initially recognized as an oncogene due to the high mutation rate in human cancer [5]. Further studies revealed P53 was an important tumor suppressor gene and critical safeguard for DNA damage, cell stress and malignant transformation process [6, 7]. P53 is composed of 393 amino acids and could be divided into three functional domains, including transcriptional activation domain, DNA binding domain and tetramerization domain [8]. The P53 protein could be activated in several conditions, such as DNA damage and oxidative stress. If it is activated, the P53 half-life will increase, which lead to enhanced activation of P53 target genes, including P21, BAX and Fas [9]. In the meantime, the activation of P53 will lead to G1-S cell cycle arrest, activation of DNA repair process or cell apoptosis. Based on the importance of P53 protein, P53 field is one of the most extensively studied genes in the cancer area. Re-activation of P53 protein is a plausible approach for cancer treatment [10].

The regulation of P53 is tightly controlled via several mechanisms, among which the post-translational modification is one of the most critical manners affecting P53 signaling activity [11-13]. In the unstressed condition, P53 is subject to continuous ubiquitination and proteasome degradation. Several E3 ubiquitin ligases were reported to promote P53 protein ubiquitination and degradation, while the most studied of these are MDM2 protein. The MDM2 protein interacts with P53 at the N-terminal and facilitates P53 ubiquitination and degradation. On the other hand, P53 could locate at the promoter region of MDM2 and facilitate MDM2 gene expression [14]. Besides, the cross regulation between MDM2 and P53, a series of RING family proteins were found to modify P53 poly-ubiquitination and degradation, which were recognized to be involved in the carcinogenic process by suppressing P53 signaling [15-17].

TRIM3 (Tripartite Motif Containing 3) belongs to the RING family proteins, which was firstly reported to associate with myosin and facilitate the target proteins transportation in cells [18]. Further studies reported that TRIM3 could function as a tumor suppressor in several cancers [19-21]. Here, our studies showed that TRIM3 correlated with good prognosis in breast cancer, but related to poor survival only in P53 wild type breast cancer patients. Molecular biology studies showed that TRIM3 promoted P53 degradation and suppressed P53 target gene expression, which ultimately promoted cancer cell growth and inhibited cisplatin-induced apoptosis in P53 wild type breast cancer cells.

\section{Materials and methods}

\section{Cell culture}

MCF-7 and HEK293 cells were ordered form American Type Culture Collection (ATCC). MCF-7 and HEK293 cells were cultured in Dulbecco's Modified Eagle's Medium that contains $4.5 \mathrm{~g} / \mathrm{L}$ glucose and $4 \mathrm{mM} \mathrm{L}$-glutamine (DMEM, 41965, Life Technologies) supplemented with 10\% Fetal Bovine Serum (FBS, 10270, Life Technologies). All cell lines were subject to cell line authentication. The cell line authentication via Short Tandem Repeat (STR) was performed via PowerPlex 21 system. The STR data of HEK293 and MCF-7 lines were found consistent with STR data in ATCC.

\section{Plasmids and siRNA}

The Flag-TRIM-3 plasmid was acquired from Origene. The HA-K48 and Ub wild type plasmids were acquired from our previous study [22]. The P53 plasmid was acquired form previous studies [16]. The TRIM3 and P53 deletion variants were sub-cloned from the original plasmids. The Lipofectamin 2000 (1662298, Invitrogen) was used for the plasmids transfection. Small interfering RNAs were used for specific gene knocking-down. The TRIM3 siRNA sequences were: CAAACGAAA GGACAACCCAdTdT; UGGGUUGUCCUUUCGUUU GdTdT and GCAACAACCAGUGUAUUCAdTdT; UGA AUACACUGGUUGUUGCdTdT. The negative control siRNA sequences were: UUCUCCGAACGUGUC ACGUTT; ACGUGACACGUUCGGAGAATT. The RNAiMAX reagent (13778150, invitrogen) was used for siRNA transfection.

\section{RNA extraction and qPCR analysis}

RNeasy plus mini kits were used to extract total RNA (Qiagen). Real-time PCR was performed as previously described [23]. 36B4 was used for internal control. The primer sequences were shown here. 36B4: F: GGCGAC CTGGAAGTCCAACT; R: CCATCAGCACCACAG CCTTC. P21: F: GTGGCTCTGATTGGCTTTCTG; R: CTGAAAACAGGCAGCCCAAG. BTG2: F: AGACGA GGCAAAGCGGTAAA; R: TCCAACCATTCACGG TCAGA. P53INP1: F: TATGCTGCCCATTTCATTT; R: CTGTGCATAACTCCTGCCCT.

\section{Quantification of cell viability}

MCF-7 cells were transfected with siTRIM3 or siControl into 24-well plates. Twenty-Four hours after transfection, the cells number was countered and 4000 cells were seeded into 96 -well plates. The relative cell viability was measured at indicated time points. Cell numbers were determined using the WST-1 cell proliferation reagent as previously described. 


\section{EdU staining assay}

For ethynly-deoxyuridine (EdU) labeled DNA, cells were incubated with EdU for $2 \mathrm{~h}$. Later on, the cells were fixed in cell culture plates with $4 \%$ formalin. The EdU positive cells were counted with statistical analysis.

\section{Flow cytometry assay}

For the cell cycle analysis, the MCF-7 cells were transfected with $50 \mathrm{uM}$ siTRIM3 or siControl. After $24 \mathrm{~h}$, cells were fixed via $70 \%$ ethanol and stained with propidium iodide. For the apoptosis assay, the MDAMB175 cells were transfected with $50 \mathrm{uM}$ siTRIM3 or siControl. Twenty-four hours post-transfection, cell were treated with $10 \mathrm{uM}$ cisplatin for $8 \mathrm{~h}$. Aftern $24 \mathrm{~h}$, cells were stained with propidium iodide and annexin $\mathrm{V}$. The BD LSR flow was used to measure the fluorescence intensity.

\section{Western blotting}

Cells were harvested and lysed with RIPA buffer. Proteins were separated by electrophoresis on SDS-polyacrylamide gel electrophoresis (PAGE) and electro-transferred to PVDF membrane. The antibodies used in this study were listed here: Anti-TRIM3 (HAP043396, Sigma); Anti-P53 (SC-126, Santa Cruz); Anti-Cleaved Caspase-3 (ab2302, Abcam); Anti-HA (MMS-101R, COVANCE); Anti-myc (9E10, ab32, Abcam); Anti-myc (Ab9106, Abcam); Anti-Actin (GB12001, Servicebio). Membranes were then washed with PBS for three times and incubated with secondary antibodies Peroxidase-Conjugated AffiniPure Goat Anti-Mouse IgG or Goat Anti-Rabbit IgG. Fluorescent signals were visualized with ECL system. (amersham imager 600, USA).

\section{Co-immunoprecipitation assay}

Immunoprecipitation was performed as described in previous study [23]. The MCF-7 cells total cell lysis was pre-cleared with rabbit IgG for $2 \mathrm{~h}$ and subsequently immunoprecipitated with TRIM3 antibody (HAP043396, Sigma) over night, while rabbit IgG (Santa Cruz) was used as the negative control. The bounded protein was analyzed by Anti-P53 antibody (SC-126, Santa Cruz). For the domain CoIP assay, the P53 or TRIM3 variants were transfected into HEK293 cells. The total cell lysis was precleared with rabbit IgG for $2 \mathrm{~h}$ and subsequently immunoprecipitated with Myc antibody overnight, while the rabbit IgG was used as the negative control. The bounded protein was analyzed via GFP antibody (Ab290, Abcam).

\section{Protein stability assays}

About $10^{5}$ MCF-7 cells were seeded into twenty-four well plates and transfected with 50 uM TRIM3 siRNA or siControl. After $48 \mathrm{~h}$, cells were treated with $100 \mathrm{uM}$ cycloheximide (C7698, Sigma) for indicated time points. Samples were subject to western blot for P53 degradation.

\section{Poly-ubiquitination detection assay}

To directly detect the enriched K48-ubiquitinated and total ubiquitinatoin P53 from the cell extracts, HEK293 cells were transfected with 0.8 ug K48 Ubi or 0.8 ug Ub plasmids together with 0.8 ug GFP-P53 plasmid and 0.8 ug Myc-TRIM3 or Myc-vector. After $24 \mathrm{~h}$, the cells were treated with $20 \mathrm{uM} \mathrm{MG132}$ for $7 \mathrm{~h}$,then total protein was extracted and pre-cleared with $30 \mathrm{ul}$ protein $\mathrm{A}$ (santa cruz, SC-2001) for $4 \mathrm{~h}$. The supernatant was collected and immunoprecipitated by P53 antibody. Western blot with HA antibody was performed to detect total or K48 poly-ubiquitinated P53.

\section{Immunofluorescence assay}

MCF-7 cells were fixed with $4 \%$ paraformaldehyde in PBS for 10 min, permeabilized with $0.2 \%$ Triton X-100 for $5 \mathrm{~min}$, and blocked by $5 \%$ BSA in PBS for $1 \mathrm{~h}$. A rabbit anti-TRIM3 polyclonal antibody (HAP043396, Sigma) and mouse anti-P53 monoclonal antibodies (SC-126, Santa Cruz) were used, followed by Alexa Flour 647 (Invitrogen) anti-rabbit antibody and FITC-conjugated anti-mouse antibodies (Jackson ImmunoResearch, West Grove, PA). As negative controls, the samples were incubated with the secondary antibodies without primary antibodies. Images were acquired under conditions fulfilling the Nyquist criterion using Nikon A+laser scanning confocal system with a 60X oil NA1.4 objective and pinhole size of 1.0 Airy Unit. The acquired pictures were further processed and assembled using ImageJ.

\section{Statistics}

Student's $t$-test, Pearson correlation coefficient, and Cox regression analysis were used for comparisons. A $P$-value of $<0.05$ was considered to be significant.

\section{Results}

TRIM3 negative correlates P53 signaling in breast cancer cells

We firstly analyzed the prognostic effect of TRIM3 in breast cancer sample. From the public available database (https://kmplot.com/analysis), we observed that TRIM3 was correlated with good overall survival in all breast cancer patients, which was consistent with previous reported role of TRIM3 in other cancers (Fig. 1a) [19-21]. However, TRIM3 related to poor prognosis especially in P53 WT breast cancer groups (Fig. 1b). Further analysis showed that TRIM3 was elevated in TP53 wild type group in breast cancer cells, but failed to correlate with 

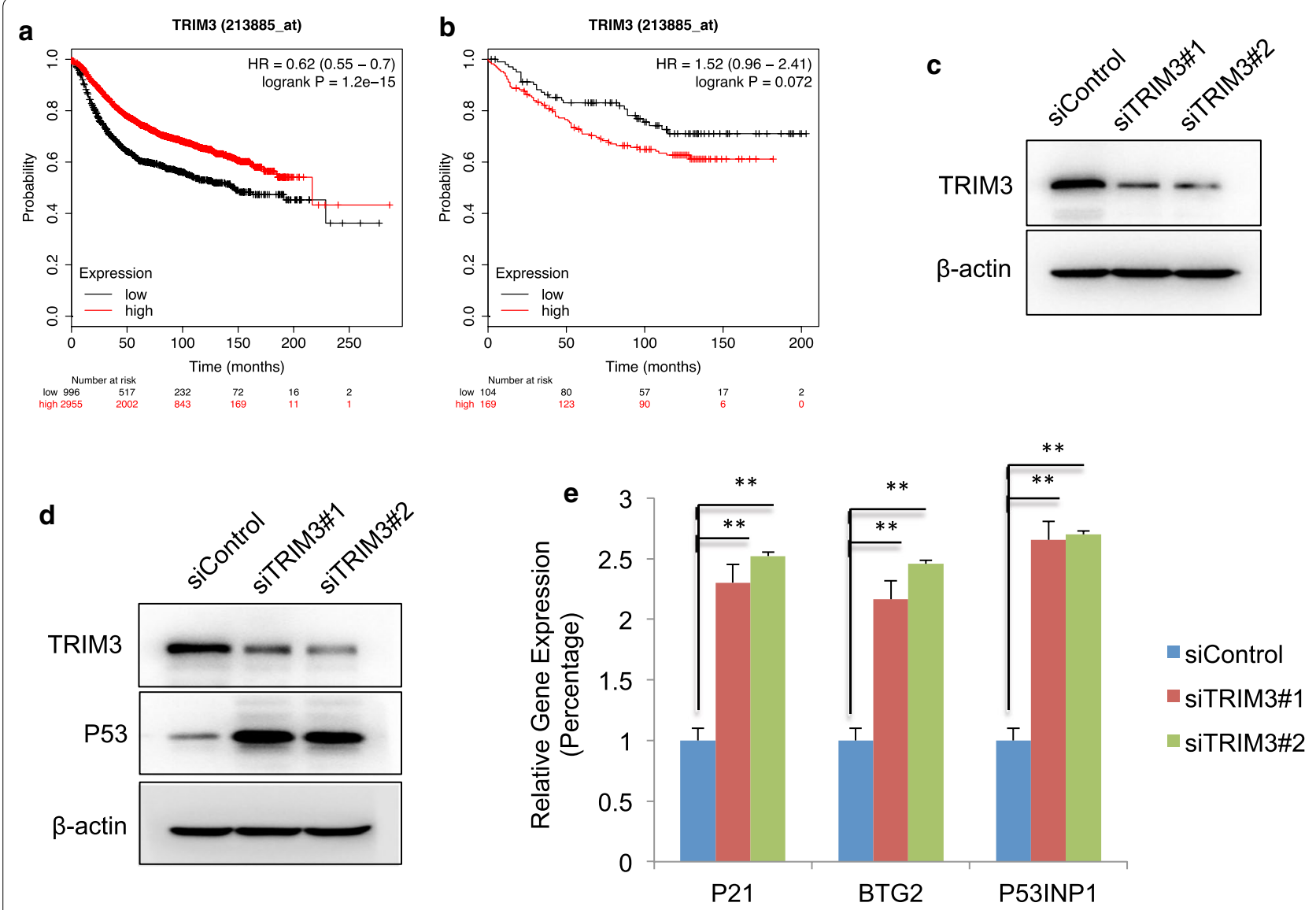

Fig. 1 TRIM3 negatively correlates P53 signaling in breast cancer cells. a The KMPLOT online analysis showed that TRIM3 related to good prognosis in all breast cancer patients. $\mathbf{b}$ The KMPLOT online analysis showed that TRIM3 tend to relate to good prognosis in P53 wild type breast cancer patients. c TRIM3 knocking down efficiency in MCF-7 cells. MCF-7 cells were transfected with TRIM3 siRNA. After 48 h, TRIM3 protein was determined by Western blot. Actin was used as internal control. d TRIM3 depletion increased P53 protein levels in MCF-7 cells. MCF-7 cells were transfected with siControl or siTRIM3. After 48 h, cells were harvested for western blot analysis. TRIM3 and P53 protein levels were determined by Western blot. Actin was used as internal control. e TRIM3 depletion increased P53 target gene expression in MCF-7 cells. MCF-7 cells were transfected with siControl or siTRIM3. After $48 \mathrm{~h}$, total RNA was extracted for gene expression analysis. Each group was done in triplicates. ${ }^{*} P<0.05$; ${ }^{* *} P<0.01$; ${ }^{* * *} P<0.001 \mathrm{for}$ target gene expression comparison

P53 target gene expression, nor prognosis in both P53 WT and mutant groups (Additional file 1: Fig. S1a-e). We further utilized MCF-7 cell, a breast cancer cell line with WT P53, as a model to carry out the cell biology studies. TRIM3 was depleted via two independent siRNAs (Fig. 1c). Immuno-blotting showed that TRIM3 depletion increased the P53 protein level in MCF-7 cells (Fig. 1d). Besides, real-time PCR assay showed that TRIM3 depletion increased classical P53 signaling target gene expression, such as CDKN1A, BTG2 and P53INP1 (Fig. 1e).

\section{TRIM3 depletion activates P53 signaling, inhibits cell} growth and promotes apoptosis in breast cancer cells In order to test the effect of TRIM3 in both normal and P53-acivated conditions, we utilized cisplatin, one chemotherapy drug, to activate P53 pathway in breast cancer cells. The immuno-blotting showed that TRIM3 could increase P53 protein level in both vehicle and cisplatin-treated conditions (Fig. 2a). Besides, Q-PCR results indicated that TRIM3 depletion increased the P53 target gene expression in both vehicle and cisplatin-treated conditions (Fig. 2b). The WST-1 assay showed that TRIM3 knocking down inhibited breast cancer cell proliferation (Fig. 2c), while the EdU staining assay also indicated TRIM3 depletion significantly decreased the cell numbers of EdU incorporation (Fig. 2d, e). We further tested the TRIM3 depletion effect in another two breast cancer cell lines with P53 WT. The data showed that TRIM3 depletion increased P53 protein level and P53 target gene expression in 


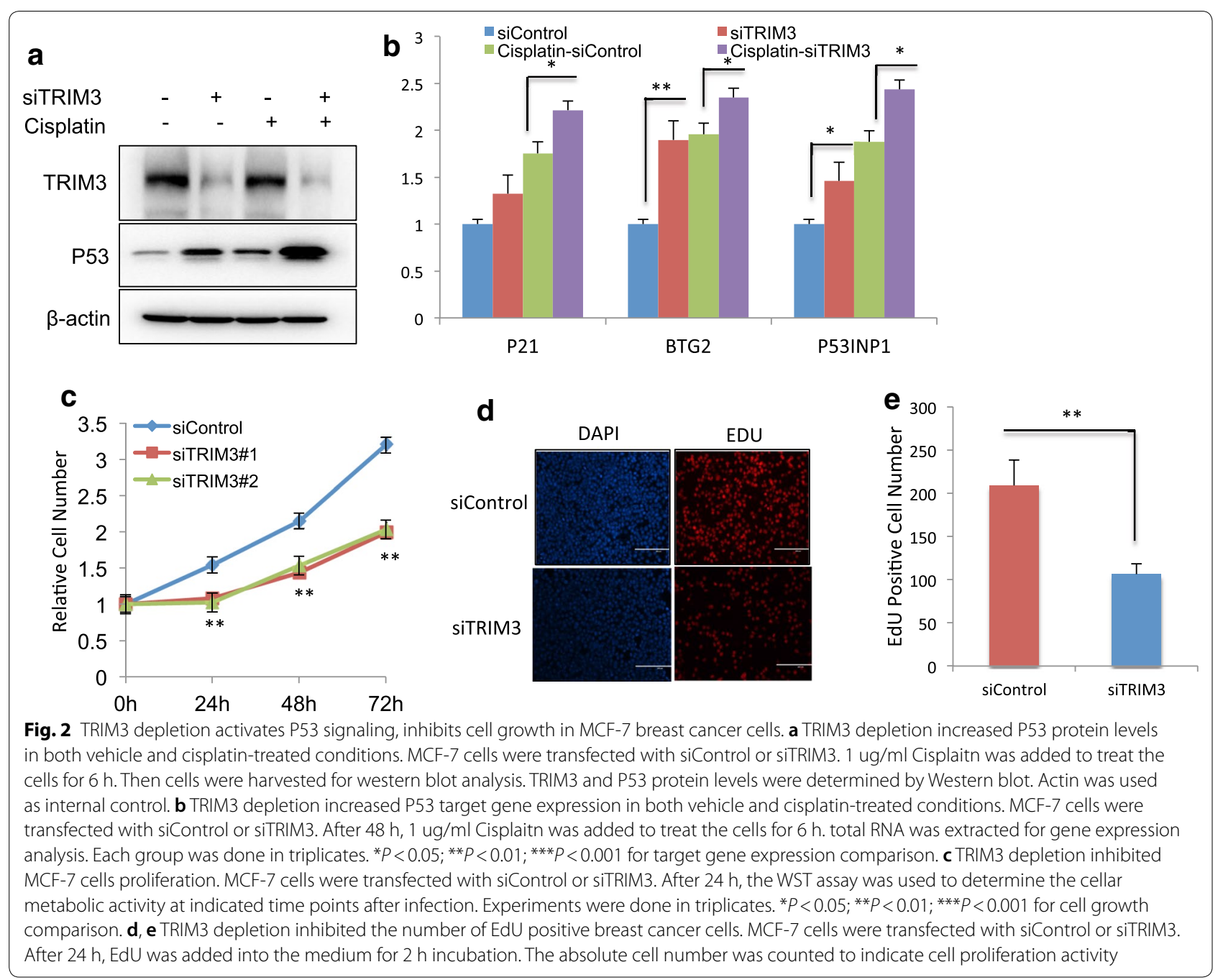

both vehicle and cisplatin-treated conditions (Figs. 3a, $\mathrm{b}$ and $4 \mathrm{a}, \mathrm{b})$. Besides, TRIM3 depletion inhibited cell proliferation in both of the two cell lines (Figs. 3c-e and $4 \mathrm{c}-\mathrm{e})$.

Since P53 is an important regulator in cell apoptosis and cell cycle, we carried out more experiments to measure TRIM3 role in cell apoptosis and cell cycle. The cell apoptosis assays were carried out mostly in MDAMB175 cells, since the apoptotic signaling is defect in MCF-7 cells. In Fig. 5a, TRIM3 depletion could significantly sensitize cisplatin-induced cell death in MDAMB175 cells. The immuno-blotting showed that TRIM3 depletion could increase cleaved-caspase 3 level, but also phospho-AKT level (Fig. 5b, c). The cell cycle analysis indicated that TRIM3 depletion could induce G1 cell cycle arrest in MCF-7 cells (Fig. 5d, e). The PI/Annexin V double staining showed that TRIM3 depletion promoted cell apoptosis in MDAMB175 cells (Fig. 5f, g).

\section{TRIM3 is localized in the cytosol and interact with P53} in breast cancer cells

We further analyzed the localization of TRIM3 and P53 in breast cancer cells. Immuno-staining showed that TRIM3 was mainly localized in the cytoplasm, while P53 was localized mostly in the nuclear (Fig. 6a). The endogenous immono-precipitation indicated that TRIM3 could associate with P53 in breast cancer cells (Fig. 6b). We further investigated the interaction domain of P53 with TRIM3. P53 is composed of three functional domains: transactivation domain, DNA binding domain and tetramerization domain (Fig. 6c), while TRIM3 is composed of four functional domains: RING domain, B1/B2 domain, CC domain and Filamin/NHL domain (Fig. 6d). We sub-cloned the variants of TRIM3 and P53. Further immuno-precipitation showed that the DNA binding domain was required for P53 to associate with TRIM3, while TRIM3 interacted with P53 via its RING domain (Fig. 6e, f). 


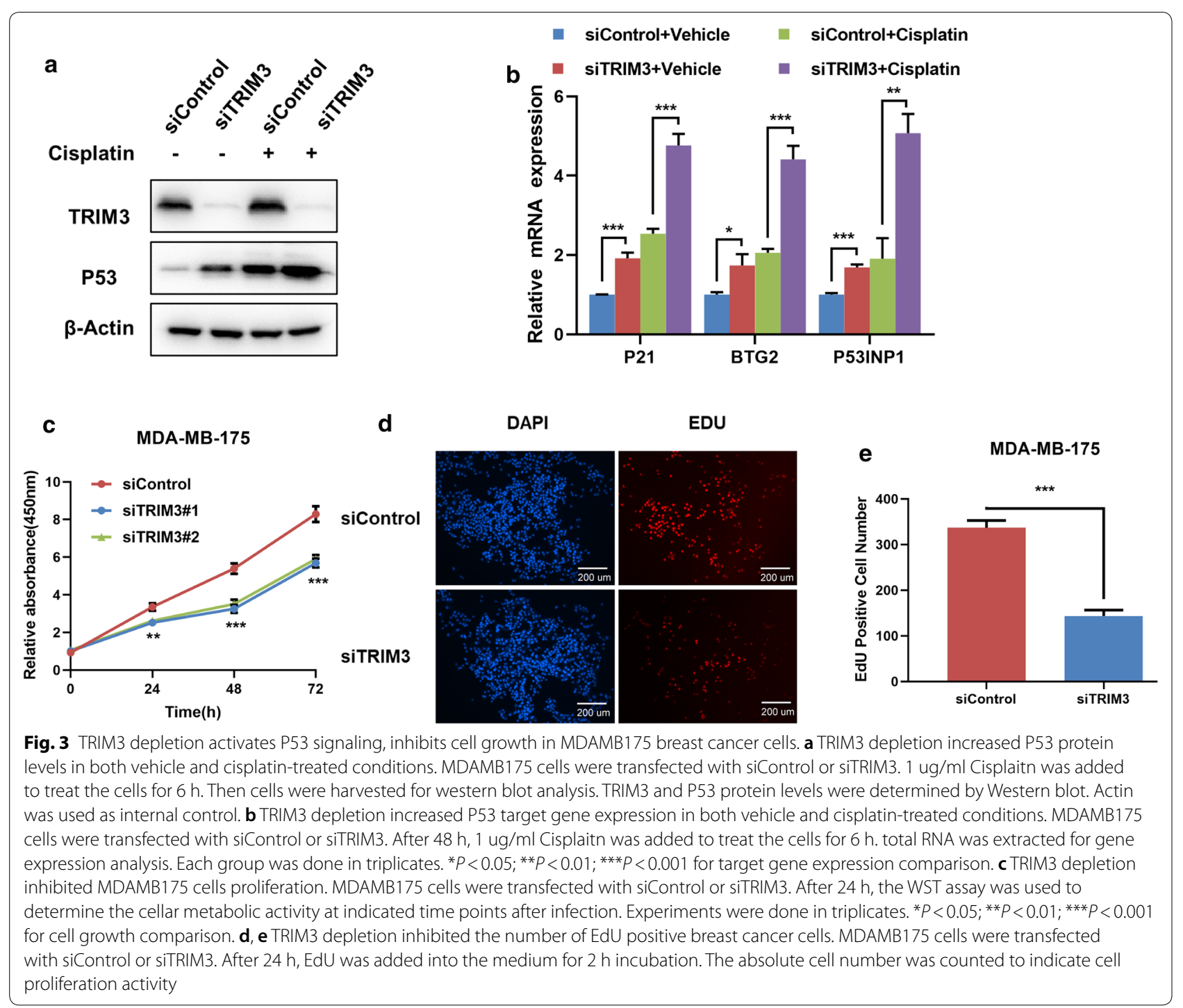

\section{TRIM3 facilitated P53 K48-linked poly-ubiquitination and degradation}

Since TRIM3 was shown to interact with P53 in breast cancer cell, we further investigated the potential molecular mechanisms. TRIM3 depletion could increase P53 protein level, which effect could be diminished via the proteasome inhibitor MG132 (Fig. 7a). This might indicated that TRIM3 modulated P53 via protein stability. Then we utilized cycloheximide, the protein synthesis inhibitor, to measure the protein stability. Figure $4 \mathrm{~b}$ showed that TRIM3 depletion significantly increased P53 half-life (Fig. 7b, c). The ubiquitin-based immuno-precipitation assay showed that TRIM3 could facilitate the overall ubiquitination level of P53 (Fig. 7d). In order to investigate the ubiquitin manner of TRIM3 on P53 protein, the K48 specific Ubiquitin plasmid were transfected to test the ubiquitination manner. The ubiquitin-based immuno-precipitation assay showed that the presence of TRIM3 could significantly promote K48linked ubiquitination of P53 (Fig. 7e).

\section{Discussion}

In the study, we demonstrated that the RING family protein TRIM3 interacted with P53 protein via its RING domain, promoted P53 K48-linked poly-ubiquitination and degradation in breast cancer cells (Fig. 8). Furthermore TRIM3 depletion induced activation of P53 signaling, cell growth arrest and cisplatin-induced apoptosis, which could be a promising therapeutic target for P53 WT breast cancer patients. 


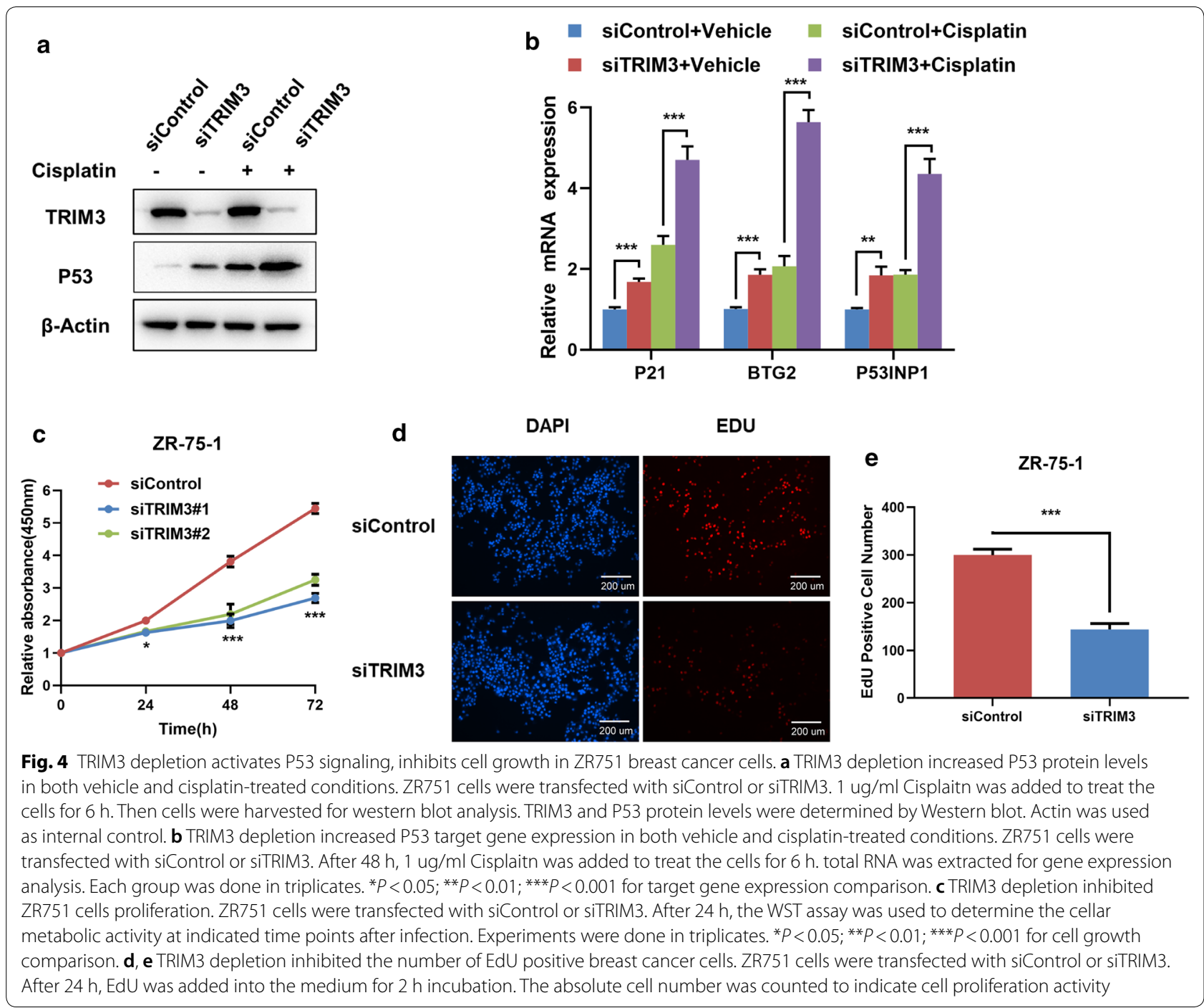

The importance of P53 in cancer has been investigated for 30 years, based on the fact that P53 is one of the most frequently mutated tumor suppressor genes in human cancer [5]. The mutated P53 could accumulate in the nuclear and disturb the assembly of DNA repair complex and facilitate tumor progression. Besides, mutated P53 could also act as an oncogene with new activities, termed "gain-of-function", that contribute to apoptosis resistance, cancer invasion and so on [24]. However, the mutation rate of P53 is approximately 30\% of all breast cancer patients [25]. Interestingly, there is a reverse correlation between ER alpha positivity and P53 mutation. The P53 mutation rate is $20 \%$ in ER alpha positive samples, while high up to $89 \%$ of P53 mutation in ER alpha negative breast cancer samples [26]. This might indicated that P53 could exert its tumor suppressor role in ER alpha positive breast cancer, which also give a possible explanation why ER alpha positive breast cancer patients have better prognosis compared with ER alpha negative ones [2]. Besides, the P53 mutations in breast cancer, P53 is also subject to several other kinds of function suppressions. For example, ER alpha could interact with P53 protein and suppress P53 target gene expression [27]. Besides, P53 expression level was decreased in breast cancer either by promoter hyper-methylation or by post-translational modification [28].

P53 is one stress-related protein with the half-life approximately $20 \mathrm{~min}$ [29]. The P53 signaling activity is precisely control mainly through ubiquitination and proteasome-dependent degradation [30]. Several E3 ubiquitin ligases have been shown to directly promote P53 poly-ubiquitination and degradation, including 


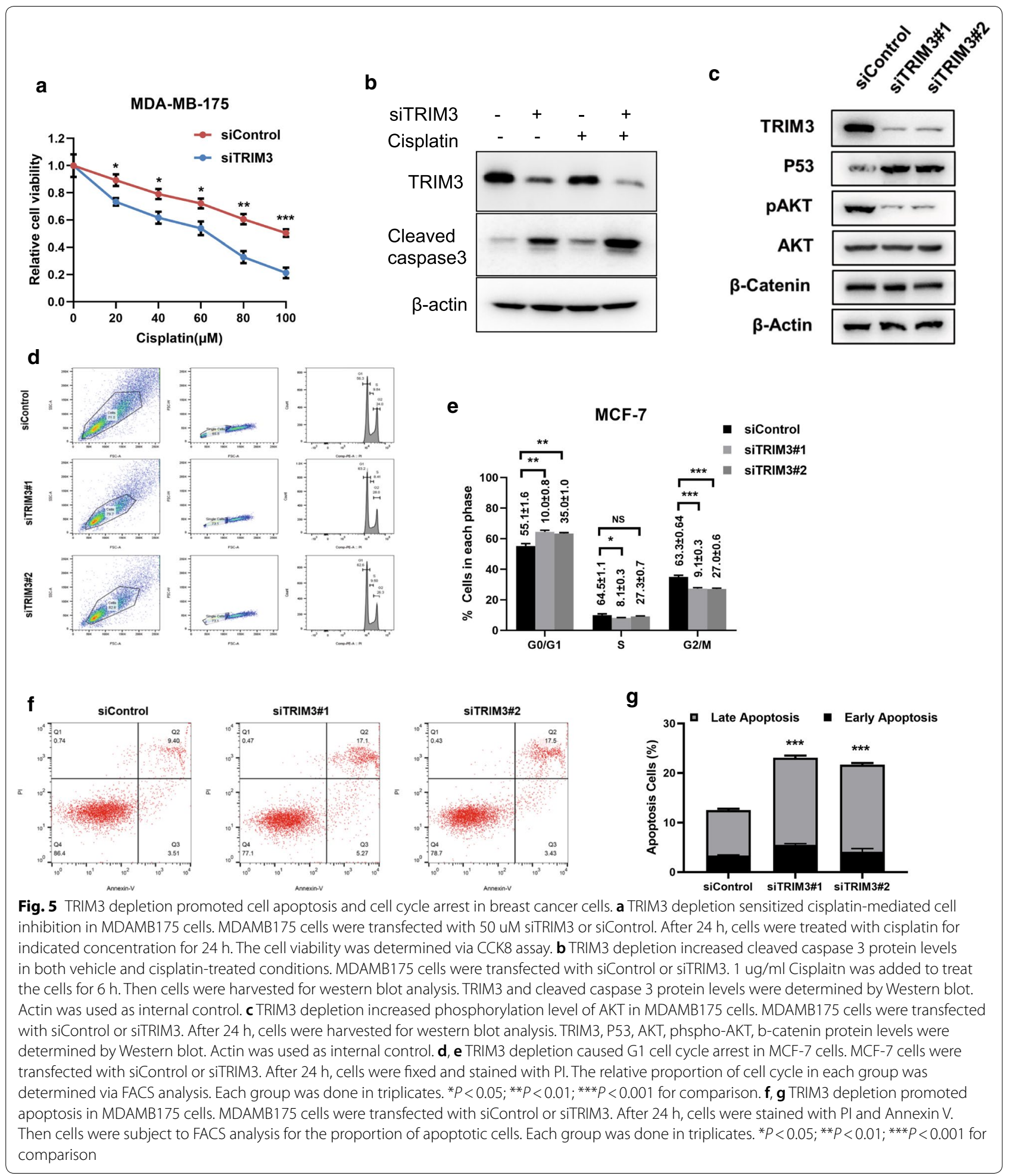

MDM2 and CHIP $[14,15]$. MDM2 is the most studied E3 ubiquitin ligase for P53 signaling. MDM2 facilitates poly-ubiquitination at several lysine residues in P53 protein and induces P53 degradation, while P53 could locate at the promoter region of MDM2 and facilitate MDM2 gene expression. However, our current study reported a novel E3 ligase TRIM3, a cytoplasmic protein, which promotes P53 ubiquitination in K48-linked 


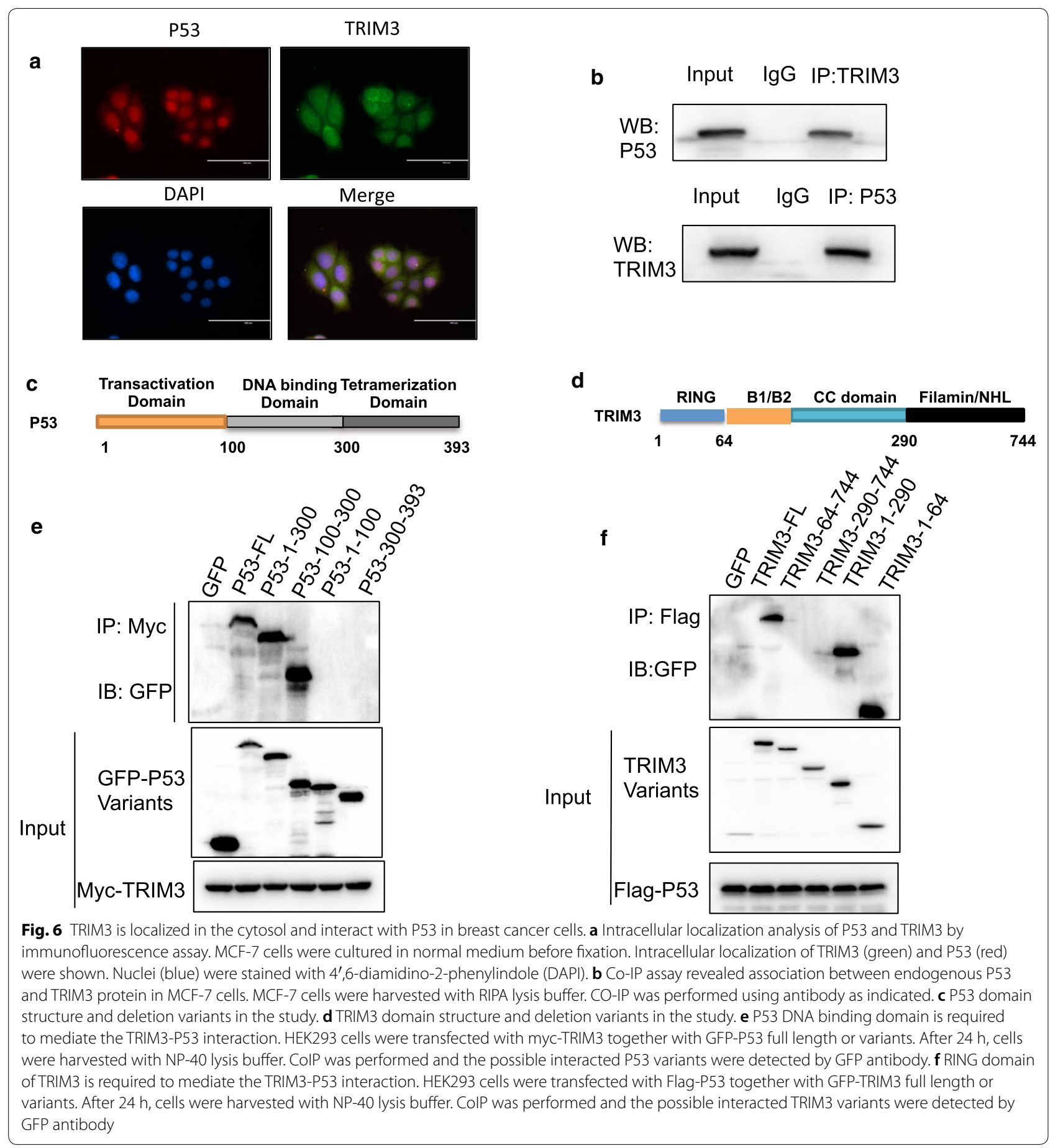

manner and degradation. Interestingly TRIM3 could interact with P53 protein, which means suppression of TRIM3 expression or small chemical compounds interfering the TRIM3-P53 interaction could be a promising strategy to restore P53 signaling activity in breast cancer cells.
TRIM3 belongs to the group of tripartite motif family and is composed of zinc-binding domain, RING domain, B1/2 domain and coiled region [31]. TIRM3 was firstly reported as the partner of myosin and facilitate the transportation of the target proteins [18]. TRIM3 were reported to be decreased in several human 


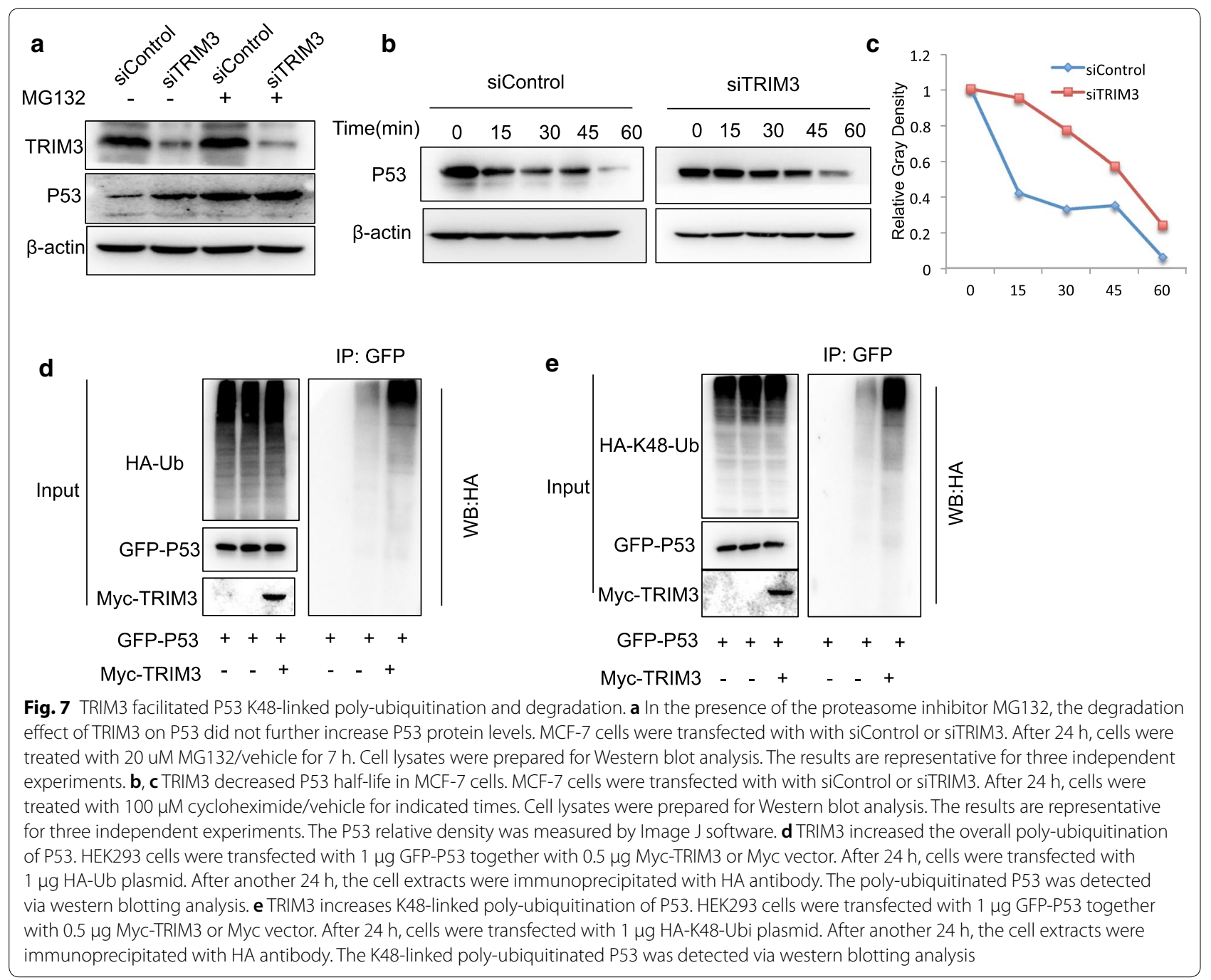

cancers, such as gastric cancer, colon cancer and liver cancer [19, 31-33]. However, although TRIM3 was shown to function as a tumor suppressor gene in several cancer types, we did not observe any dramatic TRIM3 level change in breast cancer compared with normal breast tissue, which might indicated the uncertain role or dual roles of TRIM3 might exist in breast cancer. Our molecular study revealed that TRIM3 could suppress P53 signaling, facilitate cell growth and resistance to cisplatin-induced cell apoptosis. Our finding indicates TRIM3 plays a oncogenic role in P53 WT breast cancer cells, which is opposite to previous studies [19, 31]. This interesting finding not only increases the understanding of P53 post-translational modifications but also implicates the multi-face of TRIM3 in different cancer background.
In conclusion, our study demonstrates the E3 ligase TRIM3 as a regulator of P53 signaling in human breast cancer cells. TRIM3 suppresses P53 protein level and promotes breast cancer cell growth and anti-apoptosis. As a newly discovered modulator of P53 pathway, TRIM3 could be a promising target to treat P53 WT breast cancer.

\section{Conclusion}

Our study demonstrates the E3 ligase TRIM3 as a regulator of P53 signaling in human breast cancer cells. TRIM3 suppresses P53 protein level and promotes breast cancer cell growth and anti-apoptosis. As a newly discovered modulator of P53 pathway, TRIM3 could be a promising target to treat P53 WT breast cancer. 


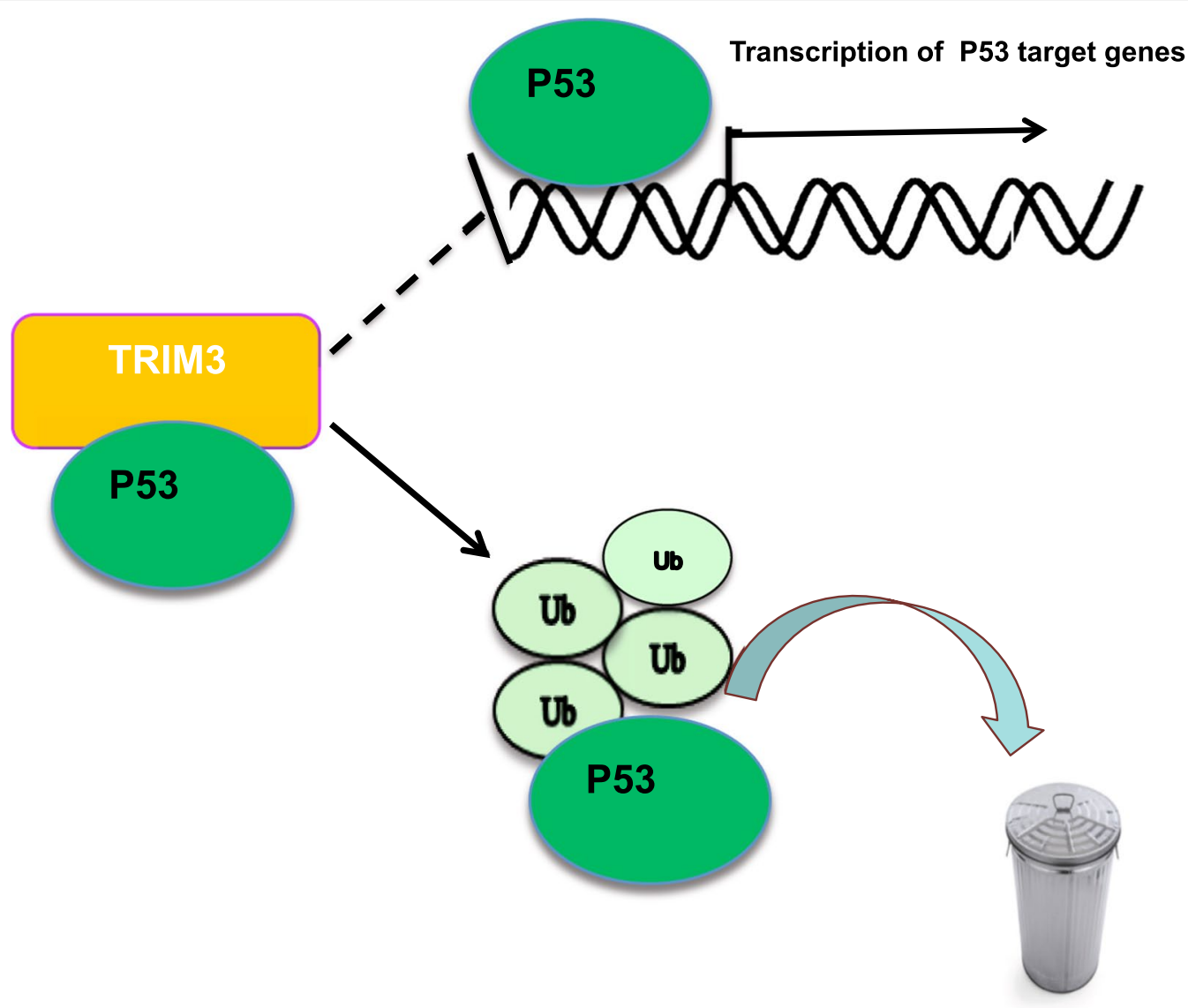

Fig. 8 The hypothetical model for TRIM3 regulating P53 pathway in P53 wild type breast cancer: TRIM3 protein associated with P53 and promoted P53 degradation via inducing P53 K48-linked poly-ubiquitination

\section{Supplementary information}

Supplementary information accompanies this paper at https://doi. org/10.1186/s12935-020-01630-z.

Additional file 1. Supplementary Figures.

\section{Abbreviations}

ER alpha: Estrogen receptor alpha; RING: Really interesting new gene; TRIM: Tripartite Motif Containing; EdU: Ethynly-deoxyuridine; MDM2: Mouse double minute 2 .

\section{Acknowledgements}

We thank all the members of Department of Breast Surgery in The First affiliated Hospital of Zhengzhou University for sharing valuable material and research support.

\section{Authors' contributions}

XW and YZ conceived the designed that study. XW, XP, GG, BX performed the molecular and cellualr biology of the study. XD and DD wrote the manuscirpt and approved the manuscirpt. All authors read and approved the final manuscript.

\section{Funding}

The project was supported from Young Scholar Grant of The First affiliated Hospital of Zhengzhou University.
Ethics approval and consent to participate

This study was reviewed and approved by the Ethical Board at The First affiliated Hospital of Zhengzhou University.

\section{Consent for publication}

Not applicable.

\section{Competing interests}

There is no competing interesting to declaim.

Received: 2 February 2020 Accepted: 29 October 2020

Published online: 23 November 2020

\section{Reference:s}

1. Bray F, et al. Global cancer statistics 2018: GLOBOCAN estimates of incidence and mortality worldwide for 36 cancers in 185 countries. CA Cancer J Clin. 2018:68(6):394-424.

2. Onitilo AA, Engel JM, Greenlee RT, Mukesh BN. Breast cancer subtypes based on ER/PR and Her2 expression: comparison of clinicopathologic features and survival. Clin Med Res. 2009;7(1-2):4-13.

3. Vaz-Luis I, et al. Outcomes by tumor subtype and treatment pattern in women with small, node-negative breast cancer: a multi-institutional study. J ClinOncol. 2014;32(20):2142-50. 
4. Shou J, et al. Mechanisms of tamoxifen resistance: increased estrogen receptor-HER2/neu cross-talk in ER/HER2-positive breast cancer. J Natl Cancer Inst. 2004;96(12):926-35.

5. Lane DP, Crawford LV. T antigen is bound to a host protein in SV40transformed cells. Nature. 1979;278(5701):261-3.

6. Mohammadzadeh A, et al. Crosstalk between P53 and DNA damage response in ageing. DNA Repair. 2019;80:8-15.

7. Thomas SE, et al. p53 and translation attenuation regulate distinct cell cycle checkpoints during endoplasmic reticulum (ER) stress. J BiolChem. 2013;288(11):7606-17.

8. Pagano B, et al. Structure and stability insights into tumour suppressor p53 evolutionary related proteins. PLoS ONE. 2013;8(10):e76014.

9. Issaeva N. p53 signaling in cancers. Cancers. 2019;11(3):332.

10. Condorelli F, Gnemmi I, Vallario A, Genazzani AA, Canonico PL. Inhibitors of histone deacetylase (HDAC) restore the $\mathrm{p} 53$ pathway in neuroblastoma cells. Br J Pharmacol. 2008;153(4):657-68.

11. Wesierska-Gadek J, Schmid G, Cerni C. ADP-ribosylation of wildtype p53 in vitro: binding of p53 protein to specific p53 consensus sequence prevents its modification. BiochemBiophys Res Commun. 1996:224(1):96-102.

12. Abraham J, Kelly J, Thibault P, Benchimol S. Post-translational modification of p53 protein in response to ionizing radiation analyzed by mass spectrometry. J MolBiol. 2000;295(4):853-64.

13. Kao CF, Chen SY, Chen JY, Wu Lee YH. Modulation of p53 transcription regulatory activity and post-translational modification by hepatitis $C$ virus core protein. Oncogene. 2004;23(14):2472-83.

14. Zhang Q, Zeng SX, Lu H. Targeting p53-MDM2-MDMX loop for cancer therapy. SubcellBiochem. 2014;85:281-319.

15. Sisoula C, Trachana V, Patterson C, Gonos ES. CHIP-dependent p53 regulation occurs specifically during cellular senescence. Free Radical Biol Med. 2011;50(1):157-65.

16. Yang $\mathrm{H}$, et al. SHARPIN facilitates $\mathrm{p} 53$ degradation in breast cancer cells. Neoplasia. 2017;19(2):84-92

17. Zhu J, et al. RING finger protein 31 promotes $\mathrm{p} 53$ degradation in breast cancer cells. Oncogene. 2016;35(15):1955-64.

18. Labonte $D$, et al. TRIM3 regulates the motility of the kinesin motor protein KIF21B. PLOS ONE. 2013;8(9):e75603.

19. Piao MY, et al. Potential role of TRIM3 as a novel tumour suppressor in colorectal cancer (CRC) development. Scand J Gastroenterol. 2016;51(5):572-82.
20. Huang $X Q$, et al. Tripartite motif-containing 3 (TRIM3) inhibits tumor growth and metastasis of liver cancer. Chin J Cancer. 2017;36(1):77.

21. Fu H, et al. Exosomal TRIM3 is a novel marker and therapy target for gastric cancer. J ExpClin Cancer Res. 2018;37(1):162.

22. Xue $M$, et al. Regulation of estrogensignaling and breast cancer proliferation by an ubiquitin ligase TRIM56. Oncogenesis. 2019;8(5):30.

23. Yang $\mathrm{H}$, et al. SMURF1 facilitates estrogen receptor a signaling in breast cancer cells. J ExpClin Cancer Res. 2018;37(1):24.

24. Duffy MJ, Synnott NC, Crown J. Mutant p53 as a target for cancer treatment. Eur J Cancer. 2017;83:258-65.

25 International Cancer Genome C, et al. International network of cancer genome projects. Nature. 2010;464(7291):993-8.

26. Miller LD, et al. An expression signature for p53 status in human breast cancer predicts mutation status, transcriptional effects, and patient survival. ProcNatlAcadSci USA. 2005;102(38):13550-5.

27. Bailey ST, Shin H, Westerling T, Liu XS, Brown M. Estrogen receptor prevents p53-dependent apoptosis in breast cancer. ProcNatlAcadSci USA. 2012;109(44):18060-5.

28. Chmelarova $\mathrm{M}$, et al. Methylation in the p53 promoter in epithelial ovarian cancer. ClinTransIOncol. 2013;15(2):160-3.

29. Wen W, et al. Knockdown of RNF2 induces apoptosis by regulating MDM2 and p53 stability. Oncogene. 2014;33(4):421-8.

30 Sane S, Rezvani K. Essential roles of E3 ubiquitin ligases in p53 regulation. Int J MolSci. 2017;18(2):442.

31. Raheja R, Liu Y, Hukkelhoven E, Yeh N, Koff A. The ability of TRIM3 to induce growth arrest depends on RING-dependent E3 ligase activity. Biochem J. 2014:458(3):537-45.

32. Chen $\mathrm{G}$, et al. Human Brat ortholog TRIM3 is a tumor suppressor that regulates asymmetric cell division in glioblastoma. Can Res. 2014;74(16):4536-48.

33. Zhu J, et al. Targeting TRIM3 deletion-induced tumor-associated lymphangiogenesis prohibits lymphatic metastasis in esophageal squamous cell carcinoma. Oncogene. 2019;38(15):2736-49.

\section{Publisher's Note}

Springer Nature remains neutral with regard to jurisdictional claims in published maps and institutional affiliations.
Ready to submit your research? Choose BMC and benefit from:

- fast, convenient online submission

- thorough peer review by experienced researchers in your field

- rapid publication on acceptance

- support for research data, including large and complex data types

- gold Open Access which fosters wider collaboration and increased citations

- maximum visibility for your research: over $100 \mathrm{M}$ website views per year

At $\mathrm{BMC}$, research is always in progress.

Learn more biomedcentral.com/submissions 